\title{
MicroRNA and gene expression changes in unruptured human cerebral aneurysms
}

\author{
Kimon Bekelis, MD, ${ }^{1}$ Joanna S. Kerley-Hamilton, PhD, ${ }^{2}$ Amy Teegarden, BS, ${ }^{3}$ \\ Craig R. Tomlinson, PhD, ${ }^{2}$ Rachael Kuintzle, BS, ${ }^{3}$ Nathan Simmons, MD, ${ }^{1,4}$ Robert J. Singer, MD, ${ }^{1,4}$ \\ David W. Roberts, MD, ${ }^{1,4}$ Manolis Kellis, PhD, ${ }^{5-7}$ and David A. Hendrix, $\mathrm{PhD}^{3,7,8}$

\begin{abstract}
${ }^{1}$ Section of Neurosurgery and 2Dartmouth Genomics and Microarray Laboratory, Dartmouth-Hitchcock Medical Center, Lebanon, New Hampshire; ${ }^{3}$ Department of Biochemistry and Biophysics and ${ }^{8}$ School of Electrical Engineering and Computer Science, Oregon State University, Corvallis, Oregon; ${ }^{4}$ Geisel School of Medicine at Dartmouth, Hanover, New Hampshire; ${ }^{5} \mathrm{MIT}$ Computational Biology Group and ${ }^{6}$ Computer Science and Artificial Intelligence Lab, MIT, Cambridge; and ${ }^{7}$ Broad Institute of MIT and Harvard, Cambridge, Massachusetts
\end{abstract}

\begin{abstract}
OBJECTIVE The molecular mechanisms behind cerebral aneurysm formation and rupture remain poorly understood. In the past decade, microRNAs (miRNAs) have been shown to be key regulators in a host of biological processes. They are noncoding RNA molecules, approximately 21 nucleotides long, that posttranscriptionally inhibit mRNAs by attenuating protein translation and promoting mRNA degradation. The miRNA and mRNA interactions and expression levels in cerebral aneurysm tissue from human subjects were profiled.
\end{abstract}

METHODS A prospective case-control study was performed on human subjects to characterize the differential expression of mRNA and miRNA in unruptured cerebral aneurysms in comparison with control tissue (healthy superficial temporal arteries [STA]). Ion Torrent was used for deep RNA sequencing. Affymetrix miRNA microarrays were used to analyze miRNA expression, whereas NanoString nCounter technology was used for validation of the identified targets.

RESULTS Overall, 7 unruptured cerebral aneurysm and 10 STA specimens were collected. Several differentially expressed genes were identified in aneurysm tissue, with MMP-13 (fold change 7.21) and various collagen genes (COL1A1, COL5A1, COL5A2) being among the most upregulated. In addition, multiple miRNAs were significantly differentially expressed, with miR-21 (fold change 16.97) being the most upregulated, and miR-143-5p (fold change -11.14) being the most downregulated. From these, miR-21, miR-143, and miR-145 had several significantly anticorrelated target genes in the cohort that are associated with smooth muscle cell function, extracellular matrix remodeling, inflammation signaling, and lipid accumulation. All these processes are crucial to the pathophysiology of cerebral aneurysms.

CONCLUSIONS This analysis identified differentially expressed genes and miRNAs in unruptured human cerebral aneurysms, suggesting the possibility of a role for miRNAs in aneurysm formation. Further investigation for their importance as therapeutic targets is needed.

http://thejns.org/doi/abs/10.3171/2015.11.JNS151841

KEY WORDS cerebral aneurysms; gene expression; microRNA expression; molecular mechanisms of disease; deep sequencing; vascular disorders

$\mathrm{C}$ EREBRAL aneurysm rupture is a devastating event with extremely high morbidity and mortality. ${ }^{32}$ The molecular mechanisms behind cerebral aneurysm formation and rupture remain poorly understood. Some investigators have attempted to identify the genetic basis of their development. These studies have focused mainly on linkage analyses, ${ }^{14,18}$ in cases of familial aneurysms, or as- sociation analyses ${ }^{40}$ of single nucleotide polymorphisms. Most recently, limited gene expression studies ${ }^{25,27,30,33-35}$ have identified genes (mainly related to inflammation and extracellular matrix remodeling) with differential expression in aneurysm tissue. These groups used microarray and polymerase chain reaction (PCR) techniques. Their results are not readily comparable due to the use of dif-

ABBREVIATIONS AAA = abdominal aortic aneurysm; $E C M$ = extracellular matrix; FDR = false discovery rate; $G O=$ Gene Ontology; miRNA = microRNA; PCR = polymerase chain reaction; $P_{C T}=$ probability of conserved target; $S T A=$ superficial temporal artery; $V S M C=$ vascular smooth muscle cell.

SUBMITTED August 6, 2015. ACCEPTED November 11, 2015.

INCLUDE WHEN CITING Published online February 26, 2016; DOI: 10.3171/2015.11.JNS151841. 
ferent microarray platforms, gene nomenclatures, and control tissues. ${ }^{34}$ While genome-wide analysis of gene expression using deep sequencing has not been performed yet, such data allow the quantification of the expression of both annotated and unannotated genes.

In the past decade, microRNAs (miRNAs) have been shown to be key regulators in a host of biological processes. ${ }^{10}$ They are noncoding RNA molecules, approximately 21 nucleotides long, that posttranscriptionally inhibit mRNAs by attenuating protein translation and promoting mRNA degradation. ${ }^{10}$ Experiments with vascular smooth muscle cell (VSMC)-specific Dicer depletion emphasized the importance of miRNAs for VSMC homeostasis, and it is therefore likely that miRNAs also play a prominent role in aneurysm formation. ${ }^{2}$ It has been shown ${ }^{2,12,17,26,28,29,31}$ that miR-29, miR-21, miR-26, miR-143, and miR-145 play pivotal roles in the formation of abdominal aortic aneurysms (AAA). These miRNAs posttranscriptionally regulate the expression of multiple targets with a function in extracellular matrix, such as collagen and fibrillin, and thus affect vascular wall stability and aneurysm formation. 2,12,17,26,28,29,31 The identification of the specific miRNAs implicated in cerebral aneurysms, and their association with gene expression, is particularly appealing because these molecules can be silenced, providing potential therapeutic targets. A recent study ${ }^{20}$ investigating the miRNA profile of ruptured cerebral aneurysms lacked controls for multiple comparisons and a separate analysis of corresponding gene expression.

To address these shortcomings, we performed a prospective case-control study in human subjects to measure the gene and miRNA expression in unruptured cerebral aneurysm tissue. Gene expression was investigated with the use of whole-genome deep sequencing. In addition, we attempted to identify statistically significant miRNAmRNA pairs with correlated expression changes that were consistent with miRNA regulation.

\section{Methods \\ Study Design}

The current study was approved by the Dartmouth Committee for the Protection of Human Subjects, and every participant signed an informed consent. We followed a prospective case-control design to investigate the expression profiles of miRNA and mRNA, as well as their correlations in unruptured human cerebral aneurysm tissue. Cerebral aneurysm samples were obtained from patients undergoing craniotomy for unruptured cerebral aneurysm clipping at our institution between 2012 and 2014.

Control tissue was collected from the superficial temporal artery (STA) of patients undergoing pterional craniotomies (for aneurysm clipping or other treatments) or patients undergoing temporal artery biopsies to rule out temporal arteritis (used only after the regular pathological analysis was negative for temporal arteritis). STA has been used in prior comparative studies of cerebral aneurysm tissue as a control. ${ }^{34}$ Clinical histories were obtained from all patients preoperatively on the day of the surgery. No postoperative follow-up data were collected for the purpose of this study.

\section{Specimen Collection}

After successful clipping of the aneurysm, the dome was resected with the use of microscissors and bayonetted forceps (not used before during the case) and was placed immediately into a vial containing RNAlater solution (Ambion) to protect RNA from degradation. The vials were immediately transported to the Genomics and Molecular Biology Shared Resource in the Geisel School of Medicine at Dartmouth, and frozen to $-80^{\circ} \mathrm{C}$. The same process was followed for the control tissue.

\section{RNA Isolation and Library Preparation}

RNA was harvested from the patient samples using the mirVana miRNA isolation kit (Ambion), which allowed total RNA that contained the miRNA population to be isolated. RNA quality and quantity were assessed using the Fragment Analyzer (Advanced Analytical) and Qubit (Invitrogen), respectively. The mRNA-sequencing libraries were prepared from $100 \mathrm{ng}$ of total RNA using the Ion Total RNA-seq kit v2 (Life Technologies).

RNA was fragmented prior to adaptor ligation, reverse transcription was performed, and the resulting cDNA was purified and amplified. This protocol allows for strand information to be preserved so that all mapped reads are aligned in the direction of transcription relative to the chromosomal strand. Each biological sample was generated with a unique INDEX sequence to allow multiple samples to be pooled together (the Ion Xpress RNA barcodes were provided by Life Technologies). Library quality was assessed using the Fragment Analyzer (Advanced Analytical) and Qubit (Invitrogen) prior to sequencing.

\section{Ion Torrent Sequencing}

RNA sequencing was performed on the Ion Proton sequencer (Life Technologies), using the PI chip, according to the manufacturer's protocol. The sequencing template was prepared from $6 \mathrm{pM}$ of the pooled libraries $(\mathrm{n}=3)$ by emulsion PCR using the One Touch 2 Instrument (Life Technologies) and the Ion PI Template OT2 200 kit v2 chemistry (Life Technologies). Following enrichment on the One Touch ES Instrument, the sample was loaded onto the PI chip and sequenced using Ion PI Sequencing v2 chemistry with the preexisting Ion RNA-whole transcriptome run plan utilizing 500 flows. The Proton server is preloaded with base-calling and adapter-trimming software. FASTQ files were generated and used for data analysis. On average (across the 17 samples), 24 million reads were generated for each barcoded biological sample, with a median read length of 90 base pairs.

The reads were aligned using TopHat (version 2.0.10), disallowing novel splice junctions, and statistically significant transcripts were identified by comparing gene expression in cerebral aneurysm and STA tissues with Cuffdiff (version 2.2.1) using each sample as a replicate and a false discovery rate (FDR) of 0.05 . Gene Ontology (GO) terms that were statistically enriched in up- and downregulated genes at this level of significance were identified with GOEAST using a hypergeometric test and a Yekutieli FDR of 0.01 . 


\section{miRNA Expression}

Affymetrix miRNA 4.0 microarrays (Affymetrix) were used to analyze the miRNA expression levels from the same RNA isolates. Following the manufacturer's protocol, 130 ng of total RNA was labeled using the FlashTag Biotin HSR RNA labeling kit (Affymetrix). To confirm efficient labeling, the ELOSA QC assay (Affymetrix) was performed, and samples were hybridized on the Affymetrix 4.0 microarrays overnight. Washing, staining, and scanning of the arrays was performed using the Command Console, and the spike-in analysis of the control oligonucleotides was evaluated using the Expression Console. Data were analyzed using the recommended Transcriptome Analysis Console software from Affymetrix.

miRNA and mRNA expression were quantified and normalized for each patient so that they could be compared across patients. We quantified the differences between cerebral aneurysms and control tissues by computing the ANOVA $p$ value for each miRNA across different individuals and using the Benjamini-Hochberg multiple test correction with an FDR of 0.15 to identify statistically significant changes in expression. An FDR of 0.15 was used to account for the inherent variability expected in clinical samples.

\section{NanoString Validation}

To validate the miRNA array expression data, the expression of approximately 800 miRNAs was determined using the NanoString nCounter Human v2 miRNA Expression Assay (NanoString Technologies) and $100 \mathrm{ng}$ of total RNA as the input. The protocol was carried out as described in the nCounter miRNA Expression Assay manual and sample preparation and hybridization protocols (November 15, 2012 version). Incubations were performed in a Veriti 96-well Thermocycler (Applied Biosystems). The duration of the overnight hybridization step was 13 hours. Samples were processed and applied to the NanoString sample cartridge using the nCounter Prep Station, and data were collected using the nCounter Digital Analyzer.

The data generated in this study were submitted to the Gene Expression Omnibus under accession number GSE66240.

\section{Results \\ Patient Characteristics}

During the study period, a total of 7 samples of unruptured cerebral aneurysm tissue (mean age 47 years; range 17-68 years; 2 male subjects) and 10 samples of control tissue (mean age 63.4 years; range 50-77 years; 1 male subject) were collected. The collected aneurysms were from the following locations: 3 in the middle cerebral artery, 2 in the posterior communicating artery, 1 in the internal carotid artery terminus, and 1 in the pericallosal artery. The mean aneurysm size was $11 \mathrm{~mm}$ (range $4-25 \mathrm{~mm}$ ).

Three of the control specimens were harvested from aneurysm patients, whose aneurysms were also collected, whereas 2 specimens belonged to aneurysm patients whose aneurysm domes were intraoperatively judged to be too small (mean size $4 \mathrm{~mm}$ ) to be safely resected. Four of the control specimens were harvested during STA bi- opsies, which eventually were deemed nonpathologic on histology. The remaining control specimen was collected during a craniotomy for tumor resection. The relative vascular comorbidities of our 2 cohorts are detailed in Supplemental Table I.

\section{Gene Expression Profile}

We identified 1028 genes (Fig. 1) with differential expression between aneurysm and control tissue at an FDR of 0.05 . From these, 623 were upregulated and 405 were downregulated. The top 20 upregulated and top 20 downregulated genes with the largest fold changes are listed in Tables 1 and 2 . The protein-coding gene with the largest fold change in expression was a member of the matrix metalloproteinase family (MMP-13) (fold change 7.21), which codes for a metalloproteinase involved in extracellular matrix (ECM) remodeling and collagenase activity. We also identified a number of host genes for miRNAs, suggesting that some of the differential expression observed in the mature miRNAs could occur before processing by Dicer and Drosha. In particular, $M I R I 43 H G$, the host gene for both miR-143 and miR-145, is downregulated in cerebral aneurysms with a highly significant 5-fold reduction, likely explaining their decreased expression. Among the most statistically enriched GO terms (Supplemental Table II) found in this data set were "extracellular matrix," "collagen metabolic process," and "response to stress."

\section{miRNA Expression Profile}

We identified 1338 miRNAs (Fig. 2) with differential expression between aneurysm and control tissue at an FDR of 0.15 . Among these, 19 had a log fold change of 2 or greater, and 5 had a log fold change of less than -2 . The 15 most upregulated and the 15 most downregulated miRNAs are presented in Table 3. The mature miRNAs with the largest absolute fold change were miR-21 (fold change 16.97) and miR-143-5p (fold change -11.14). The 3-prime product of miR-143, which has conserved target sites, was also significantly downregulated (fold change -5.27 ). Both miR-21 and miR-143 have been implicated in the pathogenesis of AAA. These results were confirmed with the use of NanoString technology, with results that were correlated with the original microarray results (Supplemental Figure I).

\section{miRNA Regulatory Effects}

We investigated our cohorts for pairs of miRNAs and their target genes that showed changes in expression that are consistent with miRNA regulation. We analyzed Targetscan Human Release 6.2, which consists of 72,770 miRNA family-gene pairs with annotated target sites in the 3' untranslated region (UTR) of at least 1 isoform. Since each miRNA family has multiple mature miRNA members, we had 165,560 distinct miRNA-gene pairs with measured expression data to investigate. Within this data set, there were 495 miRNA-gene pairs with predicted conserved target sites that satisfied both of the above-defined FDR thresholds. Furthermore, we required that the sites have a probability of conserved target $\left(\mathrm{P}_{\mathrm{CT}}\right)$ of 0.25 or greater, which resulted in 370 miR-gene pairs: $68.7 \%$ 


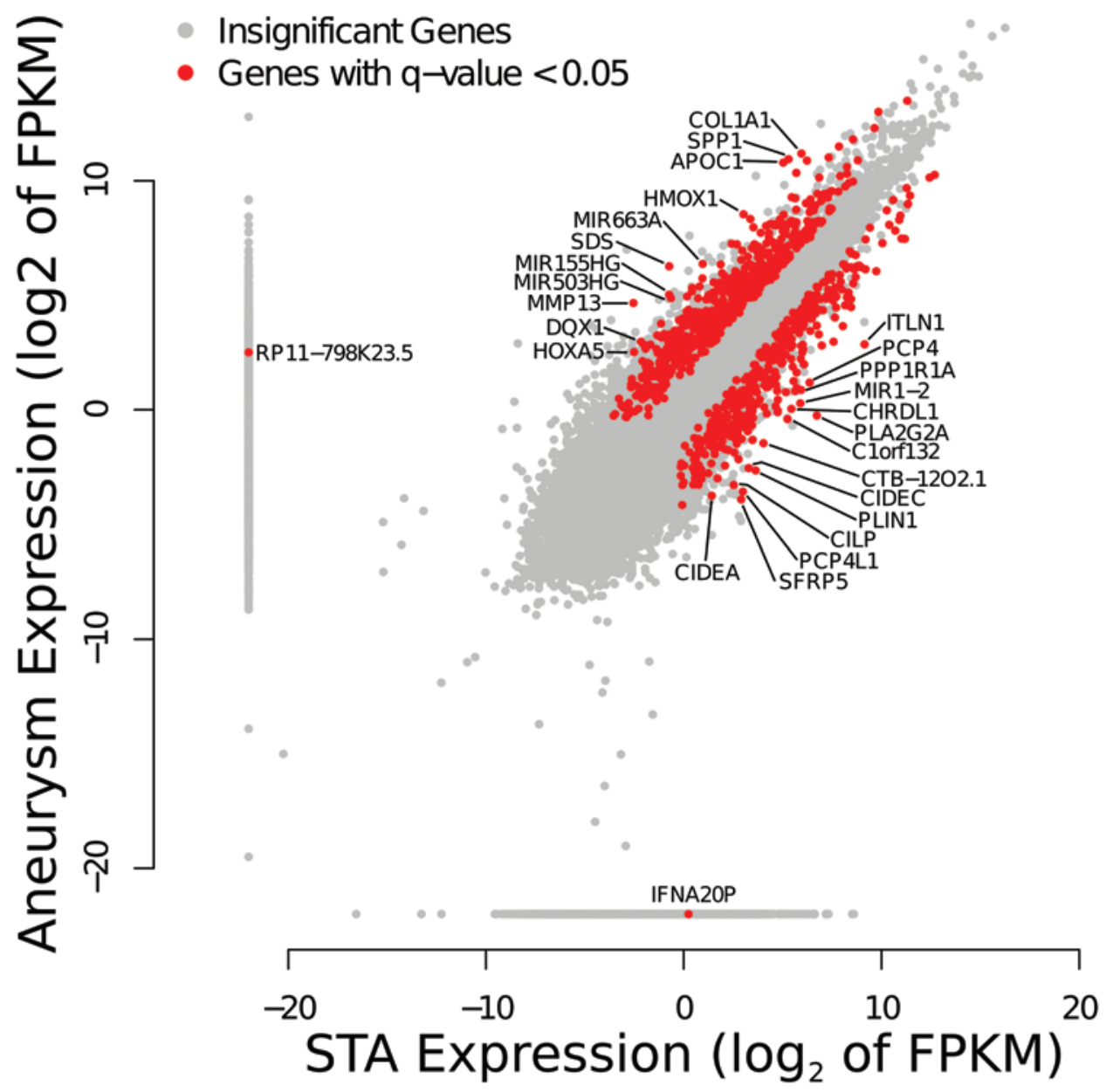

FIG. 1. Differential expression by RNA sequencing of the genes in unruptured cerebral aneurysms in comparison with STA tissues using Cuffdiff. Insignificant changes in gene expression are shown as gray dots; statistically significant changes in expression at an FDR of 0.05 are shown in red. Genes with a $\log _{2}$-fold change of 4 or greater are labeled. FPKM $=$ fragments per kilobase of transcript per million fragments mapped. Figure is available in color online only.

of which showed opposite changes in expression that were consistent with microRNA regulation. ${ }^{19}$

We then examined the effect of highly differentially expressed miRNA with a fold change of 2 or greater in this data set. Only 5 microRNAs were differentially expressed at this level, targeting 17 distinct genes (Fig. 3). Most of these miRNAs have significantly differentially expressed target genes with opposite changes in expression in comparison with miRNA. The exceptions to this pattern are miR-10b and miR-181a. Although other regulatory effects could explain these differences, it is notable that both of these miRNAs have been shown to exhibit expression that is significantly correlated with their targets..$^{16}$ Graphs demonstrating the correlation of these miRNAs with the expression of their targets are presented in Supplemental Figures II, III, IV, V, and VI.

Specifically, miR-21 was upregulated with significant downregulation of its target gene PAIP2B, a translational inhibitor. miR-143 was downregulated with the significant upregulation of several of its targets, including multiple genes involved in the metabolism of collagen (COL1A1, COL5A1, and COL5A2), MARCKS (which encodes an actin filament cross-linking protein), and TANC2. miR-145 was also downregulated with the corresponding upregulation of $A B C A 1, A D A M T S 2$, and BCAT1. The significant differential expression of miR-21, miR-143, and miR-145 was corroborated by NanoString (Supplemental Figures VII and VIII).

We additionally examined the role of the strong conservation of miRNA target sites by requiring a $\mathrm{P}_{\mathrm{CT}}$ of 0.75 or greater for the miRNA-genes pairs that matched our FDR significance thresholds. At this conservation level, $76.1 \%$ of all miRNA-gene pairs show opposite changes in expression. This list consisted of 83 target sites for 33 miRNA within 78 genes. The list of genes with 10 or more highly conserved target sites included only 5 genes, including BCAT1, TANC2, ABCA1, SIK1, and FOXF2. We found that all of the miRNAs targeting these genes showed opposite changes in expression, suggesting that the regulation of these genes could be the aggregate effect of a number of differentially expressed miRNAs. These results are summarized in Supplemental Table III.

\section{Discussion}

In this prospective study, we describe the gene and 
TABLE 1. Differentially expressed genes: Significantly upregulated genes with the highest fold change

\begin{tabular}{|c|c|c|c|c|c|c|}
\hline Gene Name & STA FPKM & $\begin{array}{c}\text { Cerebral } \\
\text { Aneurysm FPKM }\end{array}$ & $\begin{array}{l}\text { Fold Change } \\
\text { (CA/STA) }\end{array}$ & $p$ Value & Q Value & Description \\
\hline RP11-798K23.5 & 0 & 5.69579 & Inf & $5.00 \mathrm{E}-05$ & 0.00257671 & Processed pseudogene \\
\hline MMP13 & 0.171024 & 25.309 & 147.9850781 & 0.0007 & 0.0196391 & Matrix metallopeptidase-13 (collagenase-3) \\
\hline$S D S$ & 0.600028 & 77.034 & 128.3840087 & $5.00 \mathrm{E}-05$ & 0.00257671 & Serine dehydratase \\
\hline MIR155HG & 0.599021 & 32.6115 & 54.4413301 & $5.00 \mathrm{E}-05$ & 0.00257671 & MIR155 host gene (nonprotein coding) \\
\hline APOC1 & 32.6523 & 1776.04 & 54.39249302 & $5.00 \mathrm{E}-05$ & 0.00257671 & Apolipoprotein C-I \\
\hline SPP1 & 39.359 & 1972.19 & 50.10772631 & $5.00 \mathrm{E}-05$ & 0.00257671 & Secreted phosphoprotein 1 \\
\hline MIR503HG & 0.638055 & 29.3629 & 46.01938704 & 0.0026 & 0.049529 & MIR503 host gene (nonprotein coding) \\
\hline HMOX1 & 8.13042 & 371.704 & 45.7176874 & $5.00 \mathrm{E}-05$ & 0.00257671 & Heme oxygenase (decycling)-1 \\
\hline MIR663A & 1.94222 & 82.861 & 42.66303508 & $5.00 \mathrm{E}-05$ & 0.00257671 & miRNA-663a \\
\hline COL1A1 & 61.7918 & 2337.76 & 37.83285161 & $5.00 \mathrm{E}-05$ & 0.00257671 & Collagen, type I, alpha 1 \\
\hline$D Q X 1$ & 0.22374 & 7.84051 & 35.04295164 & 0.0007 & 0.0196391 & DEAQ box RNA-dependent ATPase 1 \\
\hline HOXA5 & 0.174839 & 5.74981 & 32.88631255 & 0.00185 & 0.0391237 & Homeobox A5 \\
\hline RGS1 & 10.3331 & 322.062 & 31.16799412 & $5.00 \mathrm{E}-05$ & 0.00257671 & Regulator of G-protein signaling-1 \\
\hline SLC16A10 & 0.450357 & 13.573 & 30.13831249 & 0.00015 & 0.00623662 & $\begin{array}{l}\text { Solute carrier family-16 (aromatic amino acid trans- } \\
\text { porter), member-10 }\end{array}$ \\
\hline KIAA1199 & 1.34223 & 39.8339 & 29.67740253 & $5.00 \mathrm{E}-05$ & 0.00257671 & KIAA1199 \\
\hline GREM1 & 0.249676 & 7.14698 & 28.62501802 & $5.00 \mathrm{E}-05$ & 0.00257671 & Gremlin-1, DAN family BMP antagonist \\
\hline ACP5 & 5.36724 & 152.303 & 28.37640948 & $5.00 \mathrm{E}-05$ & 0.00257671 & Acid phosphatase-5, tartrate-resistant \\
\hline APOC2 & 1.92664 & 53.409 & 27.72131794 & $5.00 \mathrm{E}-05$ & 0.00257671 & Apolipoprotein C-II \\
\hline PLA2G7 & 1.12637 & 30.951 & 27.47853725 & $5.00 \mathrm{E}-05$ & 0.00257671 & $\begin{array}{l}\text { Phospholipase-A2, group VII (platelet-activating } \\
\text { factor acetylhydrolase, plasma) }\end{array}$ \\
\hline
\end{tabular}

$\mathrm{CA}=$ cerebral aneurysm; Inf = infinity; FPKM = fragments per kilobase of transcript per million fragments mapped.

TABLE 2. Differentially expressed genes: Significantly downregulated genes with the highest absolute fold change

\begin{tabular}{|c|c|c|c|c|c|}
\hline Gene Name & STA FPKM & $\begin{array}{c}\text { Cerebral } \\
\text { Aneurysm FPKM }\end{array}$ & Log Fold & Q Value & Description \\
\hline IFNA20P & 1.18 & 0.00 & - inf & $2.26 \mathrm{E}-02$ & Interferon, alpha-20, pseudogene \\
\hline PLA2G2A & 106.23 & 0.84 & -6.98 & $2.58 \mathrm{E}-03$ & Phospholipase-A2, group IIA (platelets, synovial fluid) \\
\hline SFRP5 & 7.50 & 0.07 & -6.82 & 2.95E-02 & Secreted frizzled-related protein-5 \\
\hline PCP4L1 & 7.94 & 0.08 & -6.56 & 2.87E-02 & Purkinje cell protein 4-like-1 \\
\hline PLIN1 & 12.42 & 0.16 & -6.28 & 7.63E-03 & Perilipin 1 \\
\hline ITLN1 & 565.18 & 7.26 & -6.28 & $2.58 \mathrm{E}-03$ & Intelectin-1 (galactofuranose binding) \\
\hline CIDEC & 9.74 & 0.17 & -5.83 & $2.58 \mathrm{E}-03$ & Cell death-inducing DFFA-like effector c \\
\hline CILP & 5.76 & 0.10 & -5.81 & $2.58 \mathrm{E}-03$ & Cartilage intermediate layer protein, nucleotide pyrophosphohydrolase \\
\hline C1orf132 & 37.97 & 0.76 & -5.65 & $2.58 \mathrm{E}-03$ & Chromosome 1 open reading frame-132 \\
\hline MIR1-2 & 59.43 & 1.22 & -5.61 & $2.58 \mathrm{E}-03$ & miRNA 1-2 \\
\hline CTB-1202.1 & 16.33 & 0.36 & -5.49 & $2.58 \mathrm{E}-03$ & Uncharacterized noncoding RNA \\
\hline CHRDL1 & 43.49 & 1.03 & -5.40 & $2.58 \mathrm{E}-03$ & Chordin-like-1 \\
\hline PCP4 & 82.39 & 2.28 & -5.18 & $2.58 \mathrm{E}-03$ & Purkinje cell protein- 4 \\
\hline CIDEA & 2.67 & 0.07 & -5.17 & 1.75E-02 & Cell death-inducing DFFA-like effector a \\
\hline PPP1R1A & 61.02 & 1.83 & -5.06 & $2.58 \mathrm{E}-03$ & Protein phosphatase-1, regulatory (inhibitor) subunit $1 \mathrm{~A}$ \\
\hline FAM180B & 6.82 & 0.22 & -4.93 & $2.58 \mathrm{E}-03$ & Family with sequence similarity-180, member B \\
\hline CCL21 & 26.95 & 0.93 & -4.86 & $2.58 \mathrm{E}-03$ & Chemokine (C-C motif) ligand-21 \\
\hline SBSPON & 49.80 & 1.76 & -4.82 & $4.50 \mathrm{E}-03$ & Somatomedin B and thrombospondin, type 1 domain containing \\
\hline MIR4453 & 27.00 & 0.97 & -4.80 & $2.58 \mathrm{E}-03$ & miRNA-4453 \\
\hline AZGP1 & 11.11 & 0.40 & -4.79 & $2.58 \mathrm{E}-03$ & Alpha-2-glycoprotein-1, zinc-binding \\
\hline
\end{tabular}




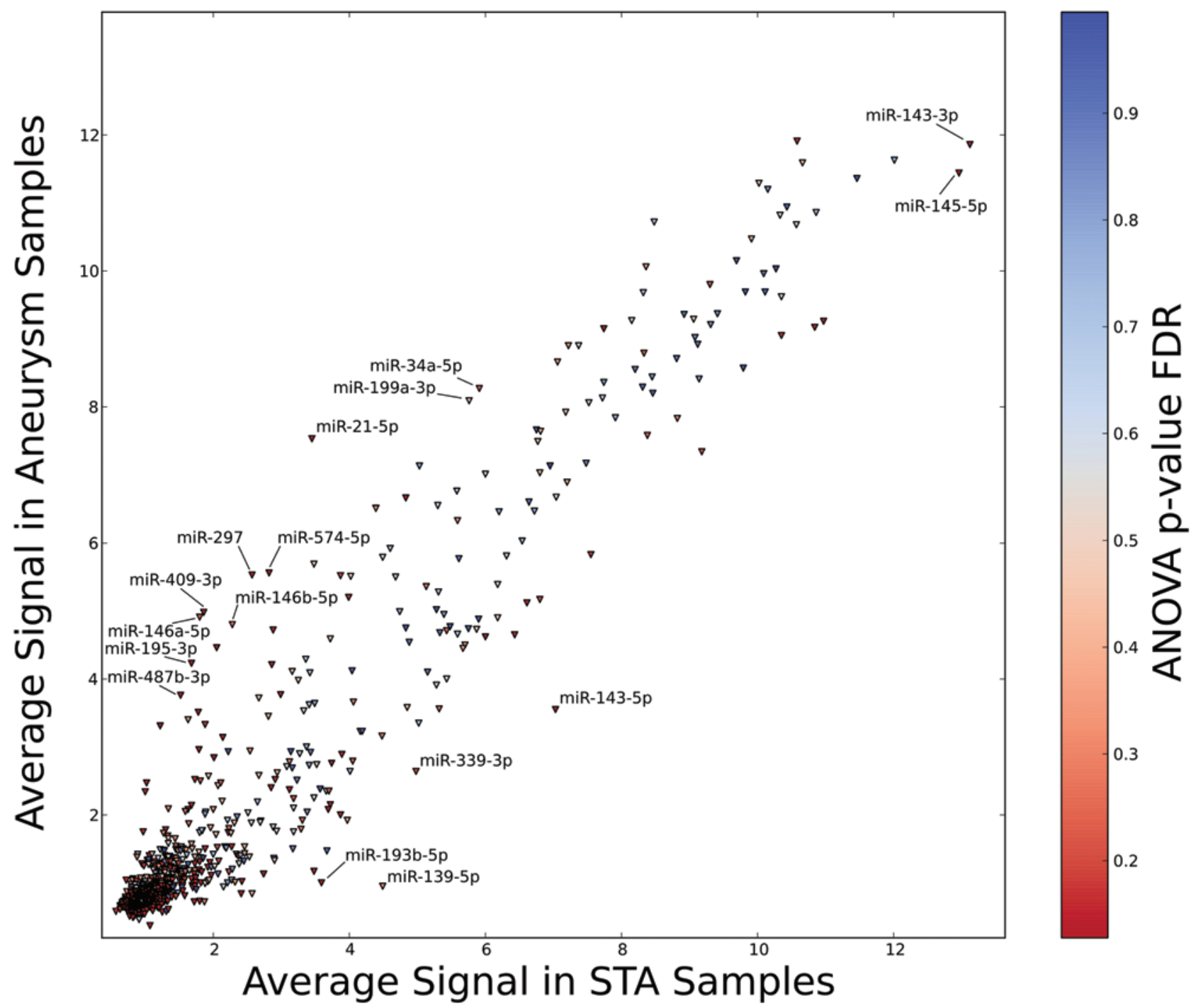

FIG. 2. Differential expression of miRNAs with annotated targets by microarray. Each triangle corresponds to a miRNA and is shaded by a color corresponding to the FDR at which it is significant. Highly significant miRNAs are labeled. Figure is available in color online only.

miRNA expression in unruptured cerebral aneurysm tissue in human subjects who underwent surgical clipping. Additionally, we detected gene-miRNA pairs with anticorrelated expression changes that were consistent with miRNA regulation. Our analysis addresses the methodological drawbacks of prior studies and utilizes deep sequencing to investigate the gene profile of unruptured human cerebral aneurysm tissue for the first time. Although changes in aneurysmal gene expression have been identified in microarray studies, the role of miRNAs in these changes is unknown. These molecules posttranscriptionally regulate mRNAs with complementary seed matches and hence could play a significant role in gene regulation. ${ }^{10}$ In addition, miRNAs are highly conserved among eukaryotic organisms, making them ideal for reverse translational research in animal models of disease.

We identified a large number of differentially expressed genes in aneurysmal tissue. The most upregulated proteincoding gene was $M M P-13$, and several members of this family have been implicated $6,7,21,22,36,37$ in the ECM remodeling of intracranial blood vessels. This results in the loss of structural support and a higher propensity for cerebral aneurysm formation. Additionally, this process can be mediated by most other highly upregulated genes in our cohort, which were involved in inflammatory regulation, ECM remodeling (SPPI), lipid metabolism $(A P O C 1)$, and collagen formation (COL1A1, COL5A1, COL5A2, and others). ${ }^{3-5,8,9,24}$ Several members of the collagen family were overexpressed, some of which have been identified in prior microarray studies. ${ }^{25,27,30,35}$ This is likely a response to the degradation of ECM and thinning of the arterial wall, resulting in fibrosis. The downregulated genes in our cohort had a complementary effect on the overexpressed ones, with potential roles in defective ECM (CILP), inadequate lipid metabolism and storage (ITLN1, PLIN1, and its target CIDEC), as well as defective apoptosis (CIDEC, SFRP5), and intracellular signaling (PLA2G2A).

The miRNA expression profiles of the cerebral an- 
TABLE 3. Significantly differentially expressed miRNAs with the highest fold changes

\begin{tabular}{|c|c|c|c|c|c|}
\hline Mature miRNA Name & Aneurysm Signal & STA Signal & Fold Change & ANOVA p Value & ANOVA FDR \\
\hline \multicolumn{6}{|l|}{ Upregulated } \\
\hline miR-21-5p & 7.53 & 3.45 & 16.97 & 0.075185 & 0.139337 \\
\hline hsa-miR-1246 & 5.86 & 2.36 & 11.26 & 0.005104 & 0.127714 \\
\hline hsa-miR-6875-3p & 5.87 & 2.43 & 10.81 & 0.001077 & 0.127714 \\
\hline hsa-miR-6753-3p & 5.59 & 2.37 & 9.28 & 0.00051 & 0.127714 \\
\hline hsa-miR-4685-3p & 5.32 & 2.17 & 8.88 & 0.000825 & 0.127714 \\
\hline hsa-miR-409-3p & 4.98 & 1.86 & 8.68 & 0.004163 & 0.127714 \\
\hline hsa-miR-297 & 5.53 & 2.57 & 7.75 & 0.000597 & 0.127714 \\
\hline hsa-miR-4484 & 8.84 & 5.98 & 7.24 & 0.000192 & 0.127714 \\
\hline hsa-miR-1972 & 4.8 & 2.03 & 6.85 & 0.000263 & 0.127714 \\
\hline hsa-miR-574-5p & 5.56 & 2.82 & 6.69 & 0.003852 & 0.127714 \\
\hline hsa-miR-6877-3p & 6.47 & 3.92 & 5.86 & 0.007492 & 0.127714 \\
\hline hsa-miR-487b-3p & 4.23 & 1.68 & 5.85 & 0.022793 & 0.127714 \\
\hline hsa-miR-619-5p & 8.87 & 6.35 & 5.72 & 0.00016 & 0.127714 \\
\hline hsa-miR-195-3p & 4.46 & 2.05 & 5.33 & 0.000684 & 0.127714 \\
\hline hsa-miR-4793-3p & 4.61 & 2.24 & 5.16 & 0.000186 & 0.127714 \\
\hline hsa-miR-4440 & 5.37 & 3.07 & 4.93 & 0.000441 & 0.127714 \\
\hline hsa-miR-382-5p & 3.76 & 1.52 & 4.72 & 0.009787 & 0.127714 \\
\hline hsa-miR-432-5p & 3.31 & 1.22 & 4.25 & 0.010858 & 0.127714 \\
\hline hsa-miR-3197 & 4.65 & 2.64 & 4.04 & 0.013788 & 0.127714 \\
\hline hsa-miR-127-3p & 6.66 & 4.83 & 3.55 & 0.014006 & 0.127714 \\
\hline \multicolumn{6}{|l|}{ Downregulated } \\
\hline hsa-miR-143-5p & 3.55 & 7.03 & 11.14 & 0.006126 & 0.127714 \\
\hline hsa-miR-3195 & 2.09 & 5.08 & -7.93 & 0.007712 & 0.127714 \\
\hline hsa-miR-6068 & 3.5 & 6.18 & -6.39 & 0.00964 & 0.127714 \\
\hline hsa-miR-193b-5p & 1 & 3.59 & -6.01 & 0.016744 & 0.127714 \\
\hline hsa-miR-6848-5p & 2.34 & 4.61 & -4.81 & 0.058811 & 0.130779 \\
\hline hsa-miR-4655-5p & 1.95 & 3.83 & -3.67 & 0.072579 & 0.137742 \\
\hline hsa-miR-606 & 2 & 3.87 & -3.64 & 0.007971 & 0.127714 \\
\hline hsa-miR-3613-3p & 10.58 & 12.39 & -3.51 & 0.014591 & 0.127714 \\
\hline hsa-miR-4445-3p & 2.47 & 4.23 & -3.39 & 0.02922 & 0.127714 \\
\hline hsa-miR-320a & 9.26 & 10.97 & -3.26 & 0.069366 & 0.135963 \\
\hline hsa-miR-5010-5p & 0.95 & 2.64 & -3.21 & 0.037616 & 0.127714 \\
\hline hsa-miR-320b & 9.17 & 10.84 & -3.19 & 0.088324 & 0.148826 \\
\hline hsa-miR-139-3p & 1.13 & 2.74 & -3.06 & 0.001682 & 0.127714 \\
\hline hsa-miR-933 & 2.08 & 3.69 & -3.06 & 0.014096 & 0.127714 \\
\hline hsa-miR-940 & 2.15 & 3.72 & -2.97 & 0.028514 & 0.127714 \\
\hline hsa-miR-452-5p & 0.84 & 2.41 & -2.96 & 0.02465 & 0.127714 \\
\hline hsa-miR-4743-5p & 1.48 & 3.02 & -2.9 & 0.072175 & 0.137684 \\
\hline hsa-miR-1468-3p & 1.73 & 3.25 & -2.86 & 0.003147 & 0.127714 \\
\hline hsa-miR-145-5p & 11.44 & 12.96 & -2.86 & 0.040347 & 0.127714 \\
\hline hsa-miR-6889-5p & 1.39 & 2.91 & -2.85 & 0.059015 & 0.130779 \\
\hline
\end{tabular}

eurysms were also investigated. To limit our analysis to functionally significant miRNA, which results in posttranscriptional mRNA degradation, we investigated our sample for anticorrelated miRNA-mRNA pairs. miR-21 demonstrated the highest expression in our cohort, and was associated with multiple downregulated target genes, with $P A I P 2 B$ (a translation repressor) being the most significant. Overexpression of miR-21 was identified before in human subjects and animal models of AAA, but not in human cerebral aneurysms.,11,23,28,38 There is controversy in regard to its role in AAA pathogenesis. Some animal AAA models ${ }^{28}$ have demonstrated that upregulation of miR-21 results in protective cellular proliferation in an attempt to repair the defective vascular wall. Others ${ }^{1,11,23,38}$ have supported that miR-21 contributes to AAA formation through the downregulation of ECM-remodeling genes. 


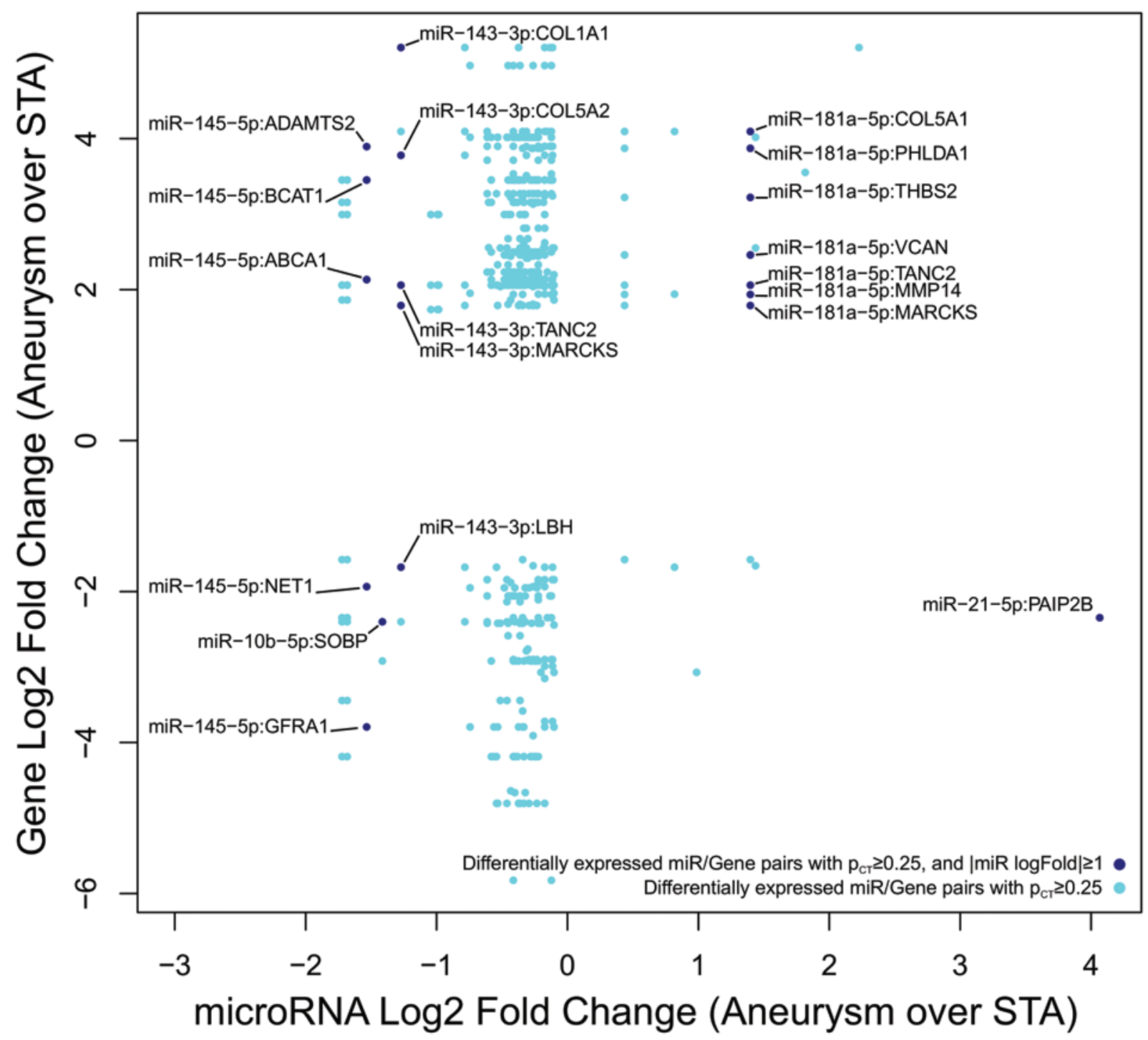

FIG. 3. The most significant miRNA-gene pairs. Each dot corresponds to a miRNA-gene pair, where dark blue corresponds to pairs with a miRNA fold change of 2 or greater, and cyan indicates all other pairs. Each column of dots with the same $x$-value corresponds to a single miRNA. At this level of significance, the majority of targeted genes have opposite changes in expression in comparison with the targeting miRNA. Figure is available in color online only.

Given the differences in pathophysiology between AAA and cerebral aneurysms, further research to unravel the role of miR-21 in the latter is needed.

miR-143 is the most significantly downregulated miRNA in our patients and has 3 upregulated target collagen genes, along with MARCKS and TANC2. Additionally, miR-143 downregulation has been identified as a central step that contributes to VSMC phenotypic modulation, which is crucial to cerebral aneurysm formation. ${ }^{11,38}$ During this process, the VSMC loses its contractile apparatus and acquires a synthetic phenotype. As a result, they dissociate from each other and migrate into the intima, producing myointimal hyperplasia. ${ }^{11,38}$ Several authors have demonstrated that this process results in the loss of structural integrity of the media, which is the layer that provides support to the vessel wall.11,38 The downregulation of miR-145 plays a complementary role in VSMC homeostasis ${ }^{13,15,17,39}$ and has been implicated in the pathophysiology of AAA in prior studies. ${ }^{11,38}$ In addition, the downregulation of miR-145 has 3 significant upregulated targets with potential involvement in the pathophysiology of cerebral aneurysms. ADAMTS2 is a metalloproteinase involved in collagen synthesis and ECM remodeling. BCAT1 contributes to cellular proliferation, and $\mathrm{ABCA} 1$ is a cholesterol efflux regulatory protein. ABCA1 and BCAT1 also had highly conserved target sites for more than 10 distinct miRNAs, which are all downregulated in expression, suggesting that other miRNAs could also play a role in their upregulation.

A prior investigation of the miRNA expression patterns by Jiang et al ${ }^{20}$ focused on ruptured cerebral aneurysms, contrary to our study that investigated only unruptured aneurysms. The generalized inflammation associated with aneurysm rupture and subarachnoid hemorrhage might mask the tissue miRNA expression patterns and make it difficult to recognize which changes contribute to inflammation and which are part of the aneurysm formation 
cascade. To address this, we elected to focus on unruptured aneurysms only. miR143 and miR145 were found to be downregulated by Jiang et al., ${ }^{20}$ in agreement with our study. However, they did not identify any upregulated miRNAs, contrary to our investigation. Jiang et al. ${ }^{20}$ did not demonstrate anticorrelated pairs of miRNA and mRNA in order to identify functionally significant miRNAs. This limitation was addressed in our study.

The current study has several limitations. First, the selection of STA as the control tissue is not ideal. Although both the STA and the intracranial vessels arise from the same parent artery (common carotid artery), extracranial vessels can potentially have different baseline genetic profiles (given the presence of adventitia) in comparison with intracranial vessels. However, collecting control tissue from normal intracranial vessels is not possible, and STA is a well-established control in the prior literature. ${ }^{34,35}$ In an attempt to further refine this control tissue, we used specimens not only from patients with aneurysms, but also from patients with unrelated intracranial pathology or no pathology at all.

Second, the present observational study design cannot establish if the identified profiles resulted in aneurysm formation or were an epiphenomenon, secondary to the presence of the aneurysm. In addition, we cannot identify the crucial molecular pathways the inhibition of which could halt or reverse aneurysm progression. However, the current data create a framework for further investigation in an animal model. The first step in this process would involve the creation of genetically modified endothelial cell lines, where the identified miRNA targets would be up- or downregulated in an effort to identify the downstream effects of these interventions and determine if the latter agree with our study. Subsequently, we would use rodent cerebral aneurysm models, where we would silence upregulated miRNAs and monitor aneurysm progression and rupture. The latter would establish if the identified targets are involved in the crucial steps of aneurysm pathophysiology and delineate the mechanism behind cerebral aneurysm progression and rupture.

Third, there is a plethora of cells that are collected and analyzed together with the aneurysm tissue, although their concentration is not expected to be different among different samples. Fourth, our results are limited to aneurysms that were large enough to allow safe resection during surgery. The molecular profiles of the smaller aneurysms might be different, and our findings do not necessarily apply to that population. Fifth, we collected aneurysms for which treatment was deemed necessary, either due to patient preference or because the aneurysm harbored some dangerous features. Though these concerns limit the generalization of our results, acquiring tissue from aneurysms that are deemed appropriate for observation is not feasible. Sixth, our sample size is limited. However, this is a preliminary single-center study, which can be used as the basis for further multicenter investigations.

\section{Conclusions}

Although there is still much work to be done to fully understand the molecular mechanisms behind cerebral an- eurysm formation and rupture, we have uncovered some likely facets of gene regulation in these tissues. Our analysis identified several significantly differentially expressed genes and miRNAs in unruptured human cerebral aneurysms. The strongest changes in expression were observed for miR-21, miR-143, miR-145, and their target genes. The majority of these genes are involved in collagen formation, inflammation regulation, lipid metabolism, smooth muscle phenotypic modification, and ECM remodeling, processes which have been implicated in cerebral aneurysm formation. Further research to separate causative and responsive gene expression differences may identify potential therapeutic targets among these miRNA-mRNA pairs.

\section{Acknowledgments}

This study was supported in part by the Dandy Fellowship Award (awarded to K.B.) by the Congress of Neurological Surgeons and by a Hitchcock Foundation Pilot Grant (K.B.) by the Hitchcock Foundation. The funders had no participation in the study design, data analysis, interpretation, or manuscript creation.

\section{References}

1. Adam M, Raaz U, Spin JM, Tsao PS: MicroRNAs in abdominal aortic aneurysm. Curr Vasc Pharmacol 13:280-290, 2015

2. Albinsson S, Suarez Y, Skoura A, Offermanns S, Miano JM, Sessa WC: MicroRNAs are necessary for vascular smooth muscle growth, differentiation, and function. Arterioscler Thromb Vasc Biol 30:1118-1126, 2010

3. Aoki T, Kataoka H, Ishibashi R, Nakagami H, Nozaki K, Morishita R, et al: Pitavastatin suppresses formation and progression of cerebral aneurysms through inhibition of the nuclear factor $\kappa B$ pathway. Neurosurgery 64:357-366, 2009

4. Aoki T, Kataoka H, Ishibashi R, Nozaki K, Hashimoto N: Cathepsin B, K, and S are expressed in cerebral aneurysms and promote the progression of cerebral aneurysms. Stroke 39:2603-2610, 2008

5. Aoki T, Kataoka H, Ishibashi R, Nozaki K, Hashimoto N: Simvastatin suppresses the progression of experimentally induced cerebral aneurysms in rats. Stroke 39:1276-1285, 2008

6. Aoki T, Kataoka H, Morimoto M, Nozaki K, Hashimoto N: Macrophage-derived matrix metalloproteinase-2 and -9 promote the progression of cerebral aneurysms in rats. Stroke 38:162-169, 2007

7. Aoki T, Kataoka H, Moriwaki T, Nozaki K, Hashimoto N: Role of TIMP-1 and TIMP-2 in the progression of cerebral aneurysms. Stroke 38:2337-2345, 2007

8. Aoki T, Kataoka H, Shimamura M, Nakagami H, Wakayama $\mathrm{K}$, Moriwaki T, et al: NF- $\kappa \mathrm{B}$ is a key mediator of cerebral aneurysm formation. Circulation 116:2830-2840, 2007

9. Aoki T, Nishimura M, Matsuoka T, Yamamoto K, Furuyashiki $\mathrm{T}$, Kataoka $\mathrm{H}$, et al: $\mathrm{PGE}_{2}-\mathrm{EP}_{2}$ signalling in endothelium is activated by haemodynamic stress and induces cerebral aneurysm through an amplifying loop via NF- $\kappa \mathrm{B}$. Br J Pharmacol 163:1237-1249, 2011

10. Bartel DP: MicroRNAs: target recognition and regulatory functions. Cell 136:215-233, 2009

11. Boon RA, Dimmeler S: MicroRNAs and aneurysm formation. Trends Cardiovasc Med 21:172-177, 2011

12. Boon RA, Seeger T, Heydt S, Fischer A, Hergenreider E, Horrevoets AJ, et al: MicroRNA-29 in aortic dilation: implications for aneurysm formation. Circ Res 109:1115-1119, 2011

13. Boucher JM, Peterson SM, Urs S, Zhang C, Liaw L: The miR-143/145 cluster is a novel transcriptional target of Jag- 
ged-1/Notch signaling in vascular smooth muscle cells. J Biol Chem 286:28312-28321, 2011

14. Broderick JP, Sauerbeck LR, Foroud T, Huston J III, Pankratz N, Meissner I, et al: The Familial Intracranial Aneurysm (FIA) study protocol. BMC Med Genet 6:17, 2005

15. Cordes KR, Sheehy NT, White MP, Berry EC, Morton SU, Muth AN, et al: miR-145 and miR-143 regulate smooth muscle cell fate and plasticity. Nature 460:705-710, 2009

16. Debernardi S, Skoulakis S, Molloy G, Chaplin T, DixonMcIver A, Young BD: MicroRNA miR-181a correlates with morphological sub-class of acute myeloid leukaemia and the expression of its target genes in global genome-wide analysis. Leukemia 21:912-916, 2007

17. Elia L, Quintavalle M, Zhang J, Contu R, Cossu L, Latronico MV, et al: The knockout of miR-143 and -145 alters smooth muscle cell maintenance and vascular homeostasis in mice: correlates with human disease. Cell Death Differ 16:1590 1598,2009

18. Foroud T, Sauerbeck L, Brown R, Anderson C, Woo D, Kleindorfer D, et al: Genome screen to detect linkage to intracranial aneurysm susceptibility genes: the Familial Intracranial Aneurysm (FIA) study. Stroke 39:1434-1440, 2008

19. Friedman RC, Farh KK, Burge CB, Bartel DP: Most mammalian mRNAs are conserved targets of microRNAs. Genome Res 19:92-105, 2009

20. Jiang Y, Zhang M, He H, Chen J, Zeng H, Li J, et al: MicroR$\mathrm{NA} / \mathrm{mRNA}$ profiling and regulatory network of intracranial aneurysm. BMC Med Genomics 6:36, 2013

21. Jin D, Sheng J, Yang X, Gao B: Matrix metalloproteinases and tissue inhibitors of metalloproteinases expression in human cerebral ruptured and unruptured aneurysm. Surg Neurol 68 (Suppl 2):S11-S16, 2007

22. Kim SC, Singh M, Huang J, Prestigiacomo CJ, Winfree CJ, Solomon RA, et al: Matrix metalloproteinase-9 in cerebral aneurysms. Neurosurgery 41:642-647, 1997

23. Kin K, Miyagawa S, Fukushima S, Shirakawa Y, Torikai K, Shimamura K, et al: Tissue- and plasma-specific MicroRNA signatures for atherosclerotic abdominal aortic aneurysm. J Am Heart Assoc 1:e000745, 2012

24. Kosierkiewicz TA, Factor SM, Dickson DW: Immunocytochemical studies of atherosclerotic lesions of cerebral berry aneurysms. J Neuropathol Exp Neurol 53:399-406, 1994

25. Krischek B, Kasuya H, Tajima A, Akagawa H, Sasaki T, Yoneyama T, et al: Network-based gene expression analysis of intracranial aneurysm tissue reveals role of antigen presenting cells. Neuroscience 154:1398-1407, 2008

26. Leeper NJ, Raiesdana A, Kojima Y, Chun HJ, Azuma J, Maegdefessel L, et al: MicroRNA-26a is a novel regulator of vascular smooth muscle cell function. J Cell Physiol 226:1035-1043, 2011

27. Li L, Yang X, Jiang F, Dusting GJ, Wu Z: Transcriptome-wide characterization of gene expression associated with unruptured intracranial aneurysms. Eur Neurol 62:330-337, 2009

28. Maegdefessel L, Azuma J, Toh R, Deng A, Merk DR, Raiesdana A, et al: MicroRNA-21 blocks abdominal aortic aneurysm development and nicotine-augmented expansion. Sci Transl Med 4:122ra22, 2012

29. Maegdefessel L, Azuma J, Toh R, Merk DR, Deng A, Chin JT, et al: Inhibition of microRNA-29b reduces murine abdominal aortic aneurysm development. J Clin Invest 122:497-506, 2012

30. Marchese E, Vignati A, Albanese A, Nucci CG, Sabatino G, Tirpakova B, et al: Comparative evaluation of genome-wide gene expression profiles in ruptured and unruptured human intracranial aneurysms. J Biol Regul Homeost Agents 24:185-195, 2010

31. Merk DR, Chin JT, Dake BA, Maegdefessel L, Miller MO, Kimura N, et al: miR-29b participates in early aneurysm development in Marfan syndrome. Circ Res 110:312-324, 2012
32. Molyneux A, Kerr R, Stratton I, Sandercock P, Clarke M, Shrimpton J, et al: International Subarachnoid Aneurysm Trial (ISAT) of neurosurgical clipping versus endovascular coiling in 2143 patients with ruptured intracranial aneurysms: a randomised trial. Lancet 360:1267-1274, 2002

33. Pera J, Korostynski M, Krzyszkowski T, Czopek J, Slowik A, Dziedzic T, et al: Gene expression profiles in human ruptured and unruptured intracranial aneurysms: what is the role of inflammation? Stroke 41:224-231, 2010

34. Roder C, Kasuya H, Harati A, Tatagiba M, Inoue I, Krischek B: Meta-analysis of microarray gene expression studies on intracranial aneurysms. Neuroscience 201:105-113, 2012

35. Shi C, Awad IA, Jafari N, Lin S, Du P, Hage ZA, et al: Genomics of human intracranial aneurysm wall. Stroke 40:1252-1261, 2009

36. Takemura Y, Hirata Y, Sakata N, Nabeshima K, Takeshita $\mathrm{M}$, Inoue T: Histopathologic characteristics of a saccular aneurysm arising in the non-branching segment of the distal middle cerebral artery. Pathol Res Pract 206:391-396, 2010

37. Tronc F, Mallat Z, Lehoux S, Wassef M, Esposito B, Tedgui A: Role of matrix metalloproteinases in blood flow-induced arterial enlargement: interaction with NO. Arterioscler Thromb Vasc Biol 20:E120-E126, 2000

38. Wei Y, Schober A, Weber C: Pathogenic arterial remodeling: the good and bad of microRNAs. Am J Physiol Heart Circ Physiol 304:H1050-H1059, 2013

39. Xin M, Small EM, Sutherland LB, Qi X, McAnally J, Plato CF, et al: MicroRNAs miR-143 and miR-145 modulate cytoskeletal dynamics and responsiveness of smooth muscle cells to injury. Genes Dev 23:2166-2178, 2009

40. Yasuno K, Bilguvar K, Bijlenga P, Low SK, Krischek B, Auburger G, et al: Genome-wide association study of intracranial aneurysm identifies three new risk loci. Nat Genet 42:420-425, 2010

\section{Disclosures}

The authors report no conflict of interest concerning the materials or methods used in this study or the findings specified in this paper.

\section{Author Contributions}

Conception and design: Bekelis. Acquisition of data: Bekelis, Kerley-Hamilton, Teegarden, Tomlinson, Kuintzle, Simmons, Singer, Roberts, Hendrix. Analysis and interpretation of data: Bekelis, Kerley-Hamilton, Teegarden, Tomlinson, Kuintzle, Kellis, Hendrix. Drafting the article: Bekelis. Critically revising the article: all authors. Reviewed submitted version of manuscript: Bekelis, Simmons, Hendrix. Approved the final version of the manuscript on behalf of all authors: Bekelis. Statistical analysis: Teegarden, Kuintzle, Kellis, Hendrix. Administrative/technical/ material support: Bekelis, Kerley-Hamilton, Tomlinson, Singer, Kellis, Hendrix.

\section{Supplemental Information Online-Only Content}

Supplemental material is available with the online version of the article.

Supplemental Figures and Tables. http://thejns.org/doi/suppl/ 10.3171/2015.11.JNS151841.

\section{Correspondence}

Kimon Bekelis, Section of Neurosurgery, Dartmouth-Hitchcock Medical Center, 1 Medical Center Dr., Lebanon, NH 03755. email: kbekelis@gmail.com. 